\title{
Expression of the transcription factor ZBTB46 distinguishes human histiocytic disorders of classical dendritic cell origin
}

\author{
Ansuman T. Satpathy $\mathbb{1}^{1,2} \cdot$ Ryanne A. Brown ${ }^{1} \cdot$ Ellen Gomulia ${ }^{1} \cdot$ Carlos G. Briseño ${ }^{3}$ - Maxwell R. Mumbach ${ }^{2,4}$. \\ Zenggang Pan $^{5} \cdot$ Kenneth M. Murphy ${ }^{3} \cdot$ Yasodha Natkunam ${ }^{1} \cdot$ Howard Y. Chang ${ }^{2,6} \cdot$ Jinah Kim ${ }^{1}$
}

Received: 12 January 2018 / Revised: 27 February 2018 / Accepted: 1 March 2018 / Published online: 9 May 2018

(c) United States \& Canadian Academy of Pathology 2018

\begin{abstract}
Distinguishing classical dendritic cells from other myeloid cell types is complicated by the shared expression of cell surface markers. ZBTB46 is a zinc finger and BTB domain-containing transcription factor, which is expressed by dendritic cells and committed dendritic cell precursors, but not by plasmacytoid dendritic cells, monocytes, macrophages, or other immune cell populations. In this study, we demonstrate that expression of ZBTB46 identifies human dendritic cell neoplasms. We examined ZBTB46 expression in a range of benign and malignant histiocytic disorders and found that ZBTB46 is able to clearly define the dendritic cell identity of many previously unclassified histiocytic disease subtypes. In particular, all examined cases of Langerhans cell histiocytosis and histiocytic sarcoma expressed ZBTB46, while all cases of blastic plasmacytoid dendritic cell neoplasm, chronic myelomonocytic leukemia, juvenile xanthogranuloma, Rosai-Dorfman disease, and Erdheim-Chester disease failed to demonstrate expression of ZBTB46. Moreover, ZBTB46 expression clarified the identity of diagnostically challenging neoplasms, such as cases of indeterminate cell histiocytosis, classifying a fraction of these entities as dendritic cell malignancies. These findings clarify the lineage origins of human histiocytic disorders and distinguish dendritic cell disorders from all other myeloid neoplasms.
\end{abstract}

\section{Key points}

- ZBTB46 expression distinguishes the dendritic cell identity of human histiocytic neoplasms.

- ZBTB46 expression is positive in Langerhans cell histiocytosis, histiocytic sarcoma, and indeterminate cell histiocytosis and negative in blastic plasmacytoid dendritic cell neoplasm, chronic myelomonocytic leukemia, juvenile xanthogranuloma, Rosai-Dorfman disease, and Erdheim-Chester disease.

These authors contributed equally: Ansuman T Satpathy, Ryanne A Brown.

Electronic supplementary material The online version of this article (https://doi.org/10.1038/s41379-018-0052-4) contains supplementary material, which is available to authorized users.

Ansuman T. Satpathy

satpathy@stanford.edu

Extended author information available on the last page of the article

\section{Introduction}

Distinguishing classical dendritic cells from other myeloid cell lineages is complicated by the shared expression of cell surface markers [1]. We previously identified Zbtb46 as a transcription factor selectively expressed by dendritic cells and dendritic cell precursors but not by related immune cell types such as plasmacytoid dendritic cells, monocytes, and macrophages $[2,3]$. Since its description, the expression of Zbtb46 has been used to accurately assign dendritic cell identity in diverse settings, including in steady-state and inflamed tissues [4-6], the tumor microenvironment [7, 8], bone marrow progenitors [2,9], and in non-model organisms [10]. Importantly, the dendritic cell-specific expression of Zbtb46 is conserved in the human immune system (as ZBTB46), nominating its use for the clinical diagnosis of dendritic cell malignancies $[2,3,11]$.

Human malignancies of dendritic cells and related myeloid cell types are often considered under the umbrella 
terms of "histiocytoses" or "histiocytic disorders" [12]. These disorders can range in clinical behavior from mild unifocal lesions, which do not require therapeutic intervention, to disseminated aggressive forms, which require systemic chemotherapy and may be fatal [12]. In contrast to the highly granular classification schemes for lymphoid malignancies and bone marrow myeloid leukemias, the categorization of histiocytic disorders is relatively unclear. One explanation for this ambiguity is the inability to accurately define the cellular identity of each histiocytic disease subtype by standard immunohistochemical methods, since surface markers are often shared between lineages. For example, while $\mathrm{B}$ and $\mathrm{T}$ cells can be clearly distinguished by their expression of $\mathrm{CD} 20$ or CD3, respectively, no analogous strategy is available to distinguish dendritic cells from macrophages. As a result, there are several non-overlapping classification schemes for histiocytic disorders currently in clinical use $[12,13]$.

Here, we developed the clinical application of an antihuman ZBTB46 antibody on formalin-fixed paraffinembedded pathology specimens to clarify the cellular identity of histiocytic disorders. We performed ZBTB46 immunohistochemistry on more than 50 primary diagnostic specimens, representing a range of benign and malignant histiocytic categories. We found that all cases of Langerhans cell histiocytosis and histiocytic sarcoma were positive for expression of ZBTB46, while blastic plasmacytoid dendritic cell neoplasm, chronic myelomonocytic leukemia, juvenile xanthogranuloma, Rosai-Dorfman disease, and Erdheim-Chester disease were negative for expression of ZBTB46. Furthermore, ZBTB46 expression clarified the identity of several diagnostically challenging cases, where the standard surface marker workup was not informative, including the identification of a subset of indeterminate cell histiocytoses as dendritic cell disorders. Since dendritic cells are continually replenished from bone marrow progenitors, while macrophages are typically seeded by embryonic progenitors and self-renew locally [1, 14], classification of histiocytic disorders as dendritic cell neoplasms may have implications for clinical therapy, for example nominating the use of bone marrow-targeted treatment strategies. In summary, these findings provide a method to accurately classify a subset of histiocytic disorders based on their lineage identity as dendritic cells, which may improve diagnostic precision and strategies for clinical therapy.

\section{Materials and methods}

\section{Case selection}

A total of 56 cases, including Langerhans cell histiocytosis (18 cases), histiocytic sarcoma (10 cases), blastic plasmacytoid dendritic cell neoplasm (7 cases), chronic myelomonocytic leukemia (4 cases), juvenile xanthogranuloma (4 cases), Rosai-Dorfman disease (3 cases), Rosai-Dorfman disease/Langerhans cell histiocytosis mixed ( 2 cases), Erdheim-Chester disease (4 cases), indeterminate cell histiocytosis ( 3 cases), and $\mathrm{S} 100+$, SOX10 + neoplasm (1 case) were identified between 2008 and 2016 in the Stanford University and University of ColoradoDenver Pathology databases. Cases were diagnosed according to the 2008 WHO Classification. In particular, cases of Langerhans cell histiocytosis were distinguished from histiocytic sarcoma by positive staining for CD1A and/or CD207. Institutional Review Board approval was obtained from Stanford University for these studies, and patient records and information were de-identified prior to analysis. One case of histiocytic sarcoma with BCL2 rearrangement was previously described [15].

\section{ZBTB46 immunohistochemistry}

Automated immunohistochemical staining was performed with Ventana Benchmark XT (Ventana Medical Systems, Inc., Tucson, AZ, USA). Four micron formalin-fixed, paraffin-embedded tissue sections were deparaffinized and rehydrated. Antigen retrieval was performed using Standard Cell Conditioning 1 (pH 8.5) pre-treatment for $60 \mathrm{~min}$ (Ventana Medical Systems). Slides were incubated with anti-human ZBTB46 (1:10, polyclonal, Sigma Aldrich, St. Louis, MO, USA) at $37^{\circ} \mathrm{C}$ for $30 \mathrm{~min}$. After primary incubation, the ultraView Universal DAB Detection system (Ventana) was used for single brown staining with 3,3' diaminobenzidine chromogen. Interpretation of staining patterns and intensity was performed by comparison to background endothelial cell staining and negative staining controls. Specifically, Zbtb46 is expressed at low levels in endothelial cells, compared to its expression in dendritic cells [2, 3]. Therefore, to guide our interpretation of ZBTB46 positivity in malignant specimens, we required ZBTB46 staining in malignant dendritic cells to be stronger than the staining observed in endothelial cells within the same section. Additional antibody staining was performed for CD1A (1:100, Clone 10, Dako \#M3571), Langerin/ CD207 (1:200, Clone 12D6, Leica - Ncl-Langerin), BRAF V600E (Clone VE1, Ventana \#790-4855), S100 (1:1000, polyclonal, Dako \#Z0311), and SOX-10 (1:50, Clone BC34, Biocare \#ACI3099C).

\section{Microarray gene expression analysis}

Microarray data (Human Genome U133 Plus 2.0 Array) for human cancer subtypes were downloaded from GEO (Supplementary Table 1). Data were quantile-normalized within each experiment and then globally scaled to adjust 


\section{a}

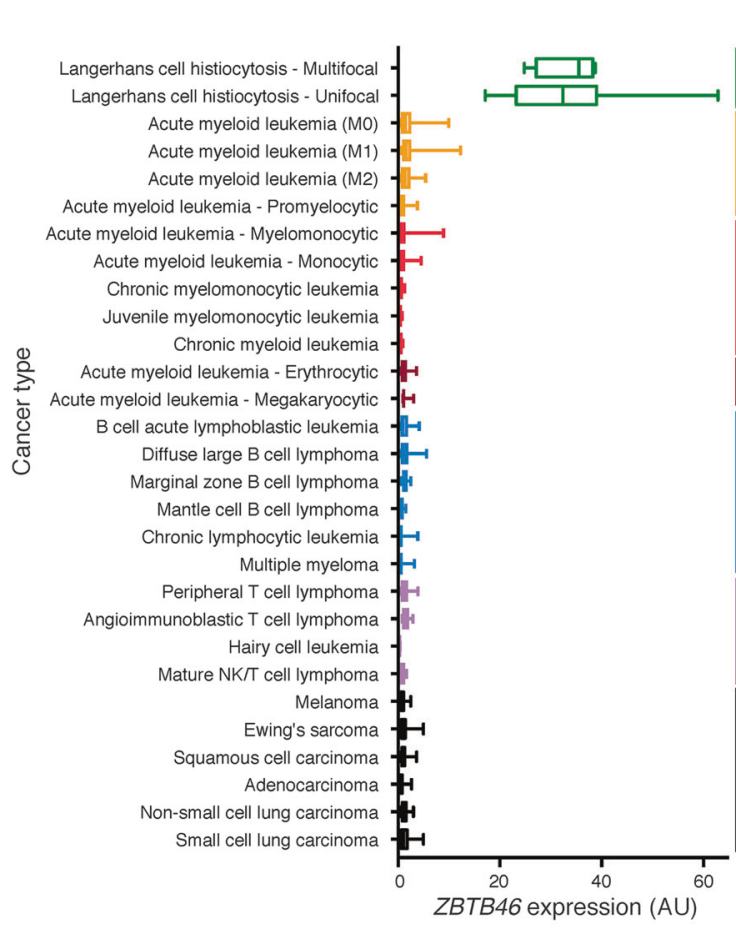

b

Cell type of origin

Dendritic cell

Hematopoietic

progenitor

Monocyte and

Granulocyte

Erythrocyte and

Megakaryocyte

B cell

T and NK cell

Non-hematopoietic

\begin{tabular}{ll}
\hline Criteria & \\
\hline Age, mean (min-max) & $20.4 \mathrm{y}(3 \mathrm{w}-73 \mathrm{y})$ \\
Gender (M/F) & $8 \mathrm{M} / 10 \mathrm{~F}$ \\
Unifocal unisystem disease & $6 / 18$ \\
Multifocal unisystem disease & $6 / 18$ \\
Multisystem disease & $6 / 18$ \\
CD1A+ / S100+ Immunohistochemistry & $18 / 18$ \\
CD207+ Immunohistochemistry & $13 / 13$ \\
BRAF V600E+ Immunohistochemistry & $4 / 7$ \\
ZBTB46+ Immunohistochemistry & $18 / 18$ \\
\hline
\end{tabular}

ZBTB46+ Immunohistochemistry $\quad 18 / 18$

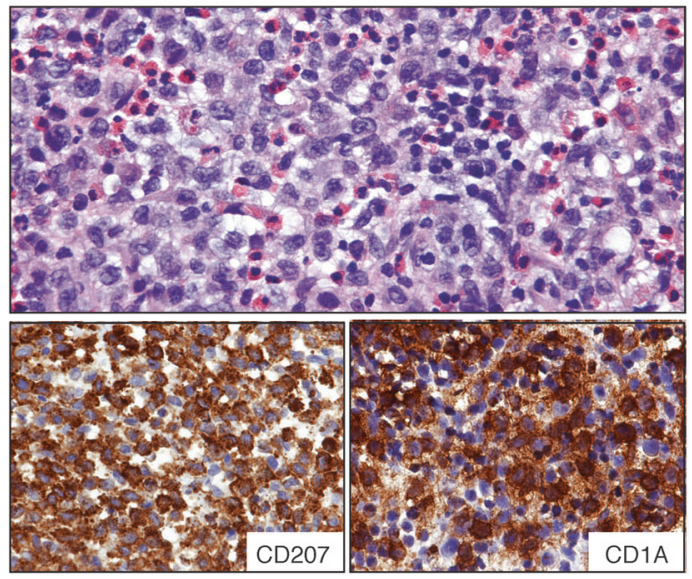

C

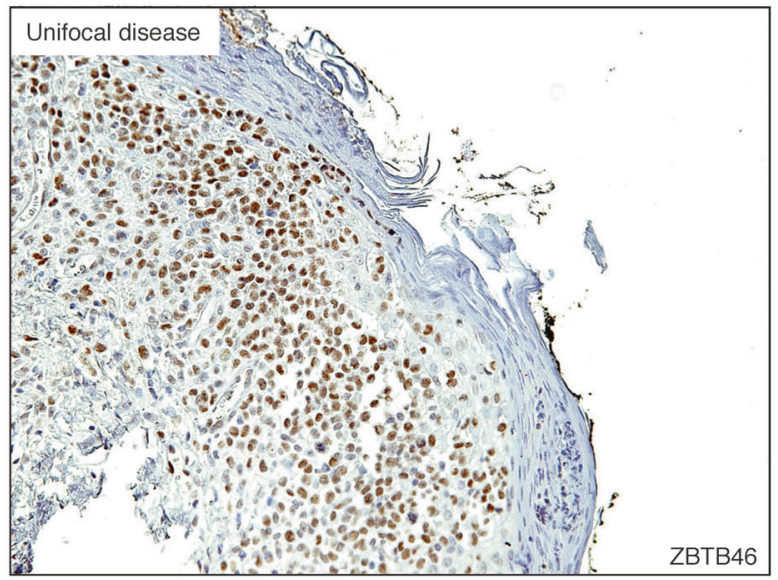

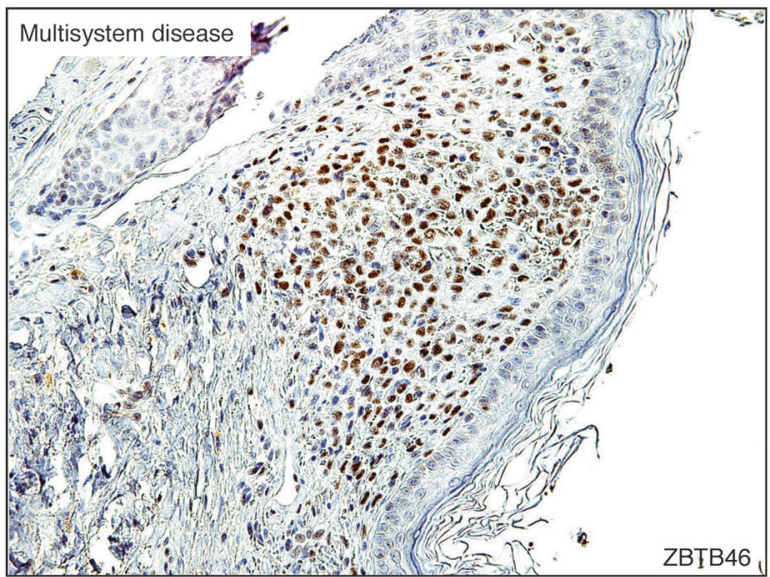

d
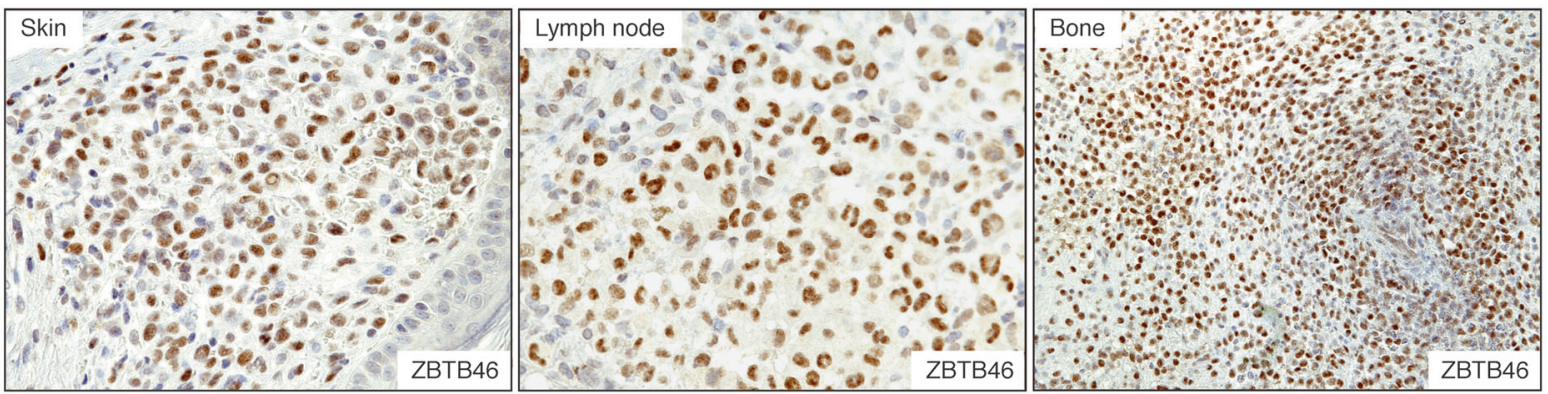

Fig. 1 ZBTB46 expression is positive in Langerhans cell histiocytosis. a $Z B T B 46$ expression in hematopoietic and non-hematopoietic cancer subtypes. For each category, the boundaries of the box represent the 75th and 25th quantiles, and whiskers represent minimum and maximum expression values across all cases. b Characteristics of Langerhans cell histiocytosis cases examined (top). H\&E (middle) and
CD207 or CD1A immunohistochemistry (bottom) of Langerhans cell histiocytosis case SU03. c ZBTB46 immunohistochemistry of representative Langerhans cell histiocytosis cases SU07 (Unifocal; left) and SU06 (Multisystem; right). d ZBTB46 immunohistochemistry of Langerhans cell histiocytosis cases in different organs (Skin: SU03, Lymph node: SU01, Bone: SU14) 
for variation between experiments (Genevestigator, ETH Zurich [16]). Cancer subtypes analyzed included Langerhans cell histiocytosis-multifocal, Langerhans cell histiocytosis-unifocal, acute myeloid leukemia, chronic myelomonocytic leukemia, juvenile myelomonocytic leukemia, chronic myeloid leukemia, B cell acute lymphoblastic leukemia, diffuse large B cell lymphoma, chronic lymphocytic leukemia, multiple myeloma, peripheral $\mathrm{T}$ cell lymphoma, angioimmunoblastic $\mathrm{T}$ cell lymphoma, hairy cell leukemia, mature NK/T cell lymphoma, melanoma, Ewing's sarcoma, squamous cell carcinoma, adenocarcinoma, non-small cell lung carcinoma, and small cell lung carcinoma.

\section{Interphase fluorescence in situ hybridization}

Fluorescence in situ hybridization (FISH) was performed on a hemodilute bone marrow aspirate specimen as previously described [15]. The BCL2 separation probe (ZytoVision \#Z2192) identifies translocation-mediated rearrangement of the BCL2 gene on chromosome 18 .

\section{Results}

To initially examine the specificity of ZBTB46 in human cancer subtypes, we analyzed RNA expression data from 5460 samples, representing 23 hematopoietic and 6 nonhematopoietic cancer subtypes (Fig. 1a, Supplementary Table 1). These data included 19 Langerhans cell histiocytosis samples from patients with uni- and multifocal disease [17]. Historically, abnormal cells in Langerhans cell histiocytosis have been thought to resemble the morphology and immunophenotype of Langerhans cells [18]. However, recent evidence from gene expression arrays and genetic mouse models has demonstrated that Langerhans cell histiocytosis may be derived from myeloid dendritic cells that express similar cell surface proteins as Langerhans cells, such as CD1A and CD207 (langerin) [17, 19-21]. Gene expression analysis showed that ZBTB46 was highly specific to Langerhans cell histiocytosis compared to closely related myeloid cancer subtypes, such as monocytic and myelomonocytic leukemia, and more broadly specific when compared to all other hematopoietic malignancies (Fig. 1a). In addition, ZBTB46 was not expressed in large-cell nonhematopoietic cancers that are often considered in the differential diagnosis of histiocytic disorders, including metastatic melanoma and carcinoma, nominating its use for distinguishing these entities in the clinical setting (Fig. 1a).

To measure ZBTB46 expression in clinical samples, we developed the use of an anti-human ZBTB46 antibody for formalin-fixed, paraffin-embedded clinical specimens. In order to validate its utility, we stained 18 cases of
Langerhans cell histiocytosis that were diagnosed at Stanford Hospital or University of Colorado-Denver between 2008 and 2016 (Supplementary Table 2). These cases represented a range of patient demographics (3 weeks old to 73 years old) and clinical phenotypes (unifocal skin lesions to multi-system disease) (Fig. 1b). Microscopically, all specimens showed collections of atypical mononuclear cells with eosinophilic cytoplasm in a background of mixed inflammation composed of variable proportions of neutrophils, eosinophils, and small lymphocytes (Fig. 1b). The atypical cells contained prominent folded or coffee-beanshaped nuclei with fine chromatin and irregular nuclear contours. All cases were positive for expression of CD1A and S100, and 4/7 tested cases were positive for VE1 antibody staining by immunohistochemistry, indicating the presence of a BRAF V600E mutation. Importantly, 18/18 Langerhans cell histiocytosis cases showed strong nuclear ZBTB46 expression in neoplastic cells (Fig. 1c, d). ZBTB46 expression was positive in Langerhans cell histiocytosis in all organ settings examined, including skin, bone, lymph node, bone marrow, and gastrointestinal tissue, demonstrating that the site of disease involvement does not impact its expression (Fig. 1d, Supplementary Table 2). These results established ZBTB46 as a nuclear marker for Langerhans cell histiocytosis and suggested the broader application of ZBTB46 immunohistochemistry for the identification of dendritic cell disorders in clinical samples.

We next analyzed ZBTB46 expression in nonLangerhans cell histiocytic disorders to identify additional subtypes with a dendritic cell phenotype. We performed ZBTB46 immunohistochemistry on 34 cases, representing the major categories of histiocytic and related disorders: blastic plasmacytoid dendritic cell neoplasm, chronic myelomonocytic leukemia, histiocytic sarcoma, juvenile xanthogranuloma, Rosai-Dorfman disease, and Erdheim-Chester disease (Supplementary Table 2). Blastic plasmacytoid dendritic cell neoplasm and chronic myelomonocytic leukemia are malignancies of plasmacytoid dendritic cells and monocytes, respectively. Both cell types are closely related to dendritic cells and derive from a common bone marrow progenitor but are negative for expression of $Z B T B 46$ in humans and mice [2, 3]. Accordingly, all cases of blastic plasmacytoid dendritic cell neoplasm and chronic myelomonocytic leukemia examined were negative for expression of ZBTB46 by immunohistochemistry (Fig. 2a). In contrast, juvenile xanthogranuloma and histiocytic sarcoma have been previously suggested to arise from dendritic cells and/or macrophages [12, 13, 22]. Both disorders are histologically similar to Langerhans cell histiocytosis, but are distinguished by key microscopic and immunophenotypic findings, including the lack of CD1A expression and Birbeck granules. However, both juvenile xanthogranuloma and histiocytic sarcoma express CD163, 
a
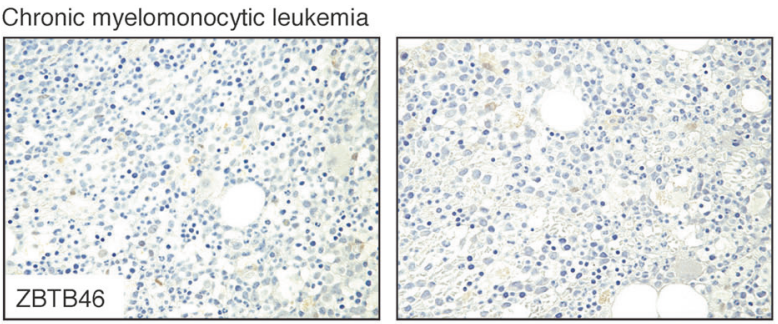

Blastic plasmacytoid dendritic cell neoplasm

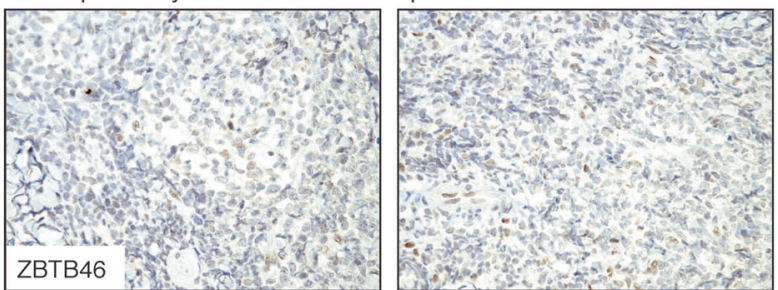

Juvenile xanthogranuloma
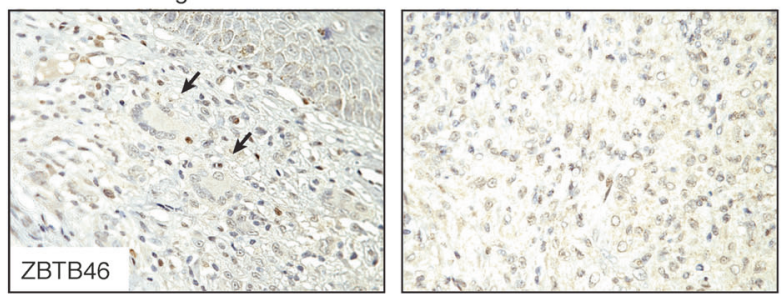

d
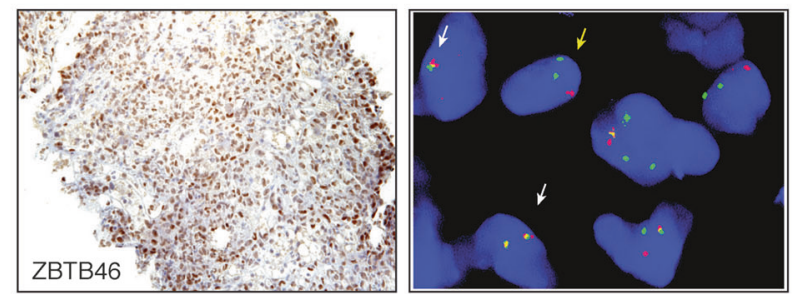

Fig. 2 ZBTB46 clarifies the dendritic cell origin of histiocytic sarcoma and indeterminate cell histiocytosis. a ZBTB46 immunohistochemistry of representative chronic myelomonocytic leukemia, blastic plasmacytoid dendritic cell neoplasm, and juvenile xanthogranuloma cases. Each image represents an individual case. Arrows indicate giant Touton cells. b Characteristics of histiocytic sarcoma cases examined (top). ZBTB46 immunohistochemistry of two representative histiocytic sarcoma cases (bottom). c Positive ZBTB46

CD68, and have been reported to harbor the BRAF V600E mutation, blurring the distinction between all three diseases [23]. Strikingly, we found that all histiocytic sarcoma cases showed strong nuclear expression of ZBTB46, while cases of juvenile xanthogranuloma were negative for expression (Fig. 2a, b). ZBTB46+ cells in histiocytic sarcoma exhibited enlarged nuclei with fine chromatin, irregular nuclear contours, and frequent mitotic figures (Fig. 2b). In contrast, neoplastic cells in juvenile xanthogranuloma were negative for ZBTB46 and demonstrated less cytologic atypia and mitotic activity. These results support a dendritic cell b

Histiocytic sarcoma case characteristics

\begin{tabular}{ll}
\hline Criteria & \\
\hline Age, mean (min-max) & $45 \mathrm{y}(9 \mathrm{~m}-89 \mathrm{y})$ \\
Gender (M/F) & $5 \mathrm{M} / 5 \mathrm{~F}$ \\
Unisystem disease & $5 / 10$ \\
Multisystem disease & $5 / 10$ \\
Coincident/history of lymphoma & $4 / 10$ \\
CD68+ Immunohistochemistry & $8 / 10$ \\
CD163+ Immunohistochemistry & $9 / 10$ \\
ZBTB46+ Immunohistochemistry & $10 / 10$ \\
\hline \hline
\end{tabular}
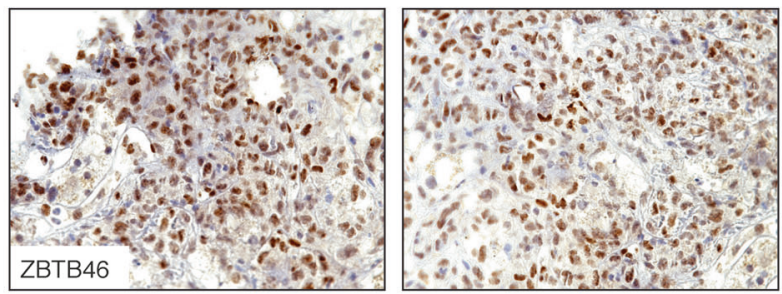

C

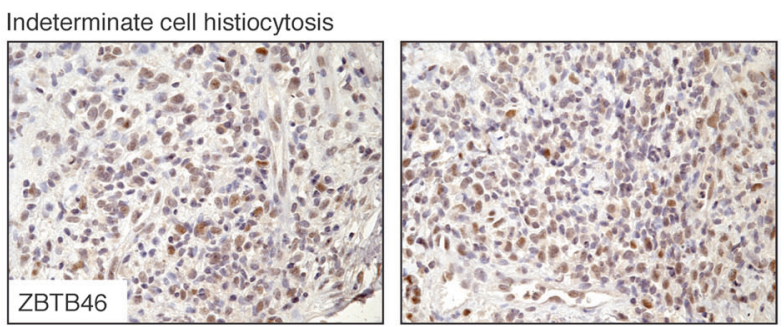

e
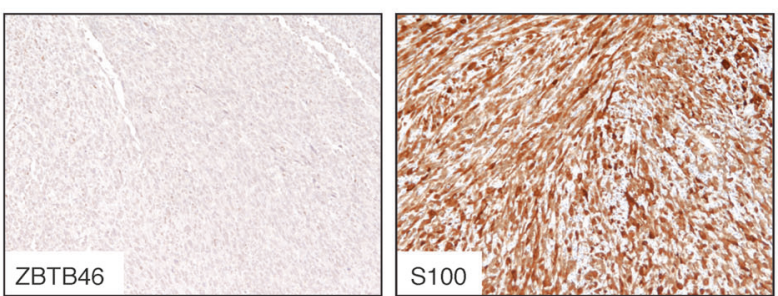

expression in two cases of indeterminate cell histiocytosis. d ZBTB46 immunohistochemistry (left) and BCL2 FISH (right) in a case with a differential diagnosis of myeloid neoplasm versus histiocytic sarcoma. Yellow arrows indicate nuclei with $B C L 2$ rearrangement, and white arrows indicate normal cells. Red dots: $5^{\prime} B C L 2$ probe, green dots: $3^{\prime}$ $B C L 2$ probe. e ZBTB46 immunohistochemistry (left) and $\mathrm{S} 100$ immunohistochemistry (right) in a case with a differential diagnosis of malignant melanoma versus dendritic cell sarcoma

phenotype of histiocytic sarcoma and macrophage phenotype of juvenile xanthogranuloma and are consistent with the aggressive, multi-organ involvement of histiocytic sarcoma and its co-occurrence with other bone marrow-derived neoplasms [24]. In line with these findings, three cases of Erdheim-Chester disease, a disease which is also thought to arise from macrophages rather than dendritic cells, were also negative for expression of ZBTB46. Finally, we assessed five cases of Rosai-Dorfman disease, two of which had coincident involvement by Langerhans cell histiocytosis, which has been observed previously [25]. ZBTB46 
expression was negative in lesional Rosai-Dorfman disease cells in all cases, but positive in Langerhans cell histiocytosis cells, demonstrating that ZBTB46 immunohistochemistry can distinguish the presence neoplastic dendritic cells from closely related myeloid cells within a single specimen (Supplementary Table 2).

We next determined whether the expression of ZBTB46 could clarify the identity of malignant cells in indeterminate cell histiocytosis. Indeterminate cell histiocytosis is a rare disorder characterized by a histiocytic infiltrate with characteristics of Langerhans cell histiocytosis and nonLangerhans cell histiocytoses. Malignant cells in indeterminate cell histiocytosis variably express markers consistent with Langerhans cells/dendritic cells (CD1A, S-100) and monocytes/macrophages (factor XIIIa, CD68), but are distinguished from malignant cells in Langerhans cell histiocytosis by their lack of Birbeck granules by electron microscopy and expression of CD207 (langerin) by immunohistochemistry, and in some cases by the presence of an ETV3-NCOA2 translocation [26, 27]. These characteristics have made it challenging to determine: (1) the cellular identity of malignant cells in indeterminate cell histiocytosis, and (2) whether indeterminate cell histiocytosis is a single disease entity, or consists of several morphologically overlapping diseases. We performed ZBTB46 immunohistochemistry in three cases of indeterminate cell histiocytosis, each of which demonstrated dermal infiltrates of mononuclear histiocytes expressing CD1A and CD68. Surprisingly, $2 / 3$ cases showed positive nuclear expression for ZBTB46 in the malignant histiocytes, while 1/3 showed no expression (Fig. 2c). These results suggest that at least a subset of indeterminate cell histiocytosis cases contains malignant dendritic cells and supports further investigation of molecular heterogeneity within this classification of histiocytic disease.

Finally, we asked whether ZBTB46 immunohistochemistry could aid in the clarification of differential diagnoses for tumors that lacked hallmark disease-associated surface markers. We first analyzed ZBTB46 expression in an undefined myeloid neoplasm infiltrating the liver (Fig. 2d). Histologically, the liver biopsy showed infiltration by sheets of CD45+ large atypical cells, but markers for myeloid subsets were negative, including CD1A, S100, CD123 (a marker for blastic plasmacytoid dendritic cell neoplasm), CD68, and CD163. However, we found strong nuclear staining of ZBTB46 in the malignant population, suggesting a dendritic cell tumor (Fig. 2d). Analysis of bone marrow infiltrates showed involvement by the same population of atypical myeloid cells and the presence of a coincident follicular lymphoma [15]. In addition, atypical cells in the bone marrow and liver possessed a complex BCL2 rearrangement: a $5^{\prime} / 3^{\prime} B C L 2$ signal separation pattern was observed in 18/200 (9\%) nuclei (Fig. 2d). Altogether, these findings supported the classification of this neoplasm as histiocytic sarcoma with $B C L 2$ rearrangement $[15,28]$. Conversely, the absence of ZBTB46 expression in clinical specimens was also useful in eliminating histiocytic disorders from the differential diagnosis. We tested ZBTB46 expression in a SOX10+/S100+ neoplasm for which the histologic findings were consistent with either recurrent malignant melanoma or a dendritic cell sarcoma (Fig. 2e). Interestingly, atypical cells in this specimen did not express hallmark markers for either melanoma (HMB45, Tyrosinase, MITF, Melanin) or histiocytic sarcoma (CD68, CD163). However, the absence of ZBTB46 expression supported its classification as recurrent malignant melanoma (Fig. 2e).

\section{Discussion}

In summary, we demonstrate the clinical utility of ZBTB46 expression as an indicator of dendritic cell lineage identity in malignant histiocytic disorders. We show that the inclusion of ZBTB46 immunohistochemistry in the work-up of histiocytic disorders clarifies the classification of these disease subtypes, particularly in cases where typical hallmark surface markers are not expressed. ZBTB46 expression was positive in all cases of Langerhans cell histiocytosis and histiocytic sarcoma examined, and in a subset of cases of indeterminate cell histiocytosis, while all other hematopoietic neoplasms and one case of melanoma (with an immunohistochemical profile indistinguishable from that of a dendritic cell neoplasm) tested were negative, indicating the high sensitivity and specificity of this marker. In neoplasms that expressed ZBTB46, it was difficult to identify co-expressed cell surface markers that reliably reflected dendritic cell lineage, since distinct disease entities often demonstrated overlapping immunohistochemical findings. But generally, expression of S100 or CD207/langerin was correlated with dendritic cell identity, with the latter marker being specific for Langerhans cell histiocytosis, while CD163 expression was correlated with macrophage identity. CD68 was non-specific and expressed across a variety of histiocytic neoplasms.

Future studies should focus on determining the diagnostic specificity and utility of this reagent across additional malignant and non-malignant specimens. In this study, one setting where ZBTB46 immunohistochemistry was particularly useful was in a case of histiocytic sarcoma with ambiguous immunophenotype and a clonal relationship to coincident follicular lymphoma [15]. The potential utility of ZBTB46 in this setting is supported by prior studies, which demonstrated that histiocytic/dendritic cell neoplasms arising from other hematolymphoid malignancies, including follicular lymphoma and chronic lymphocytic leukemia, 


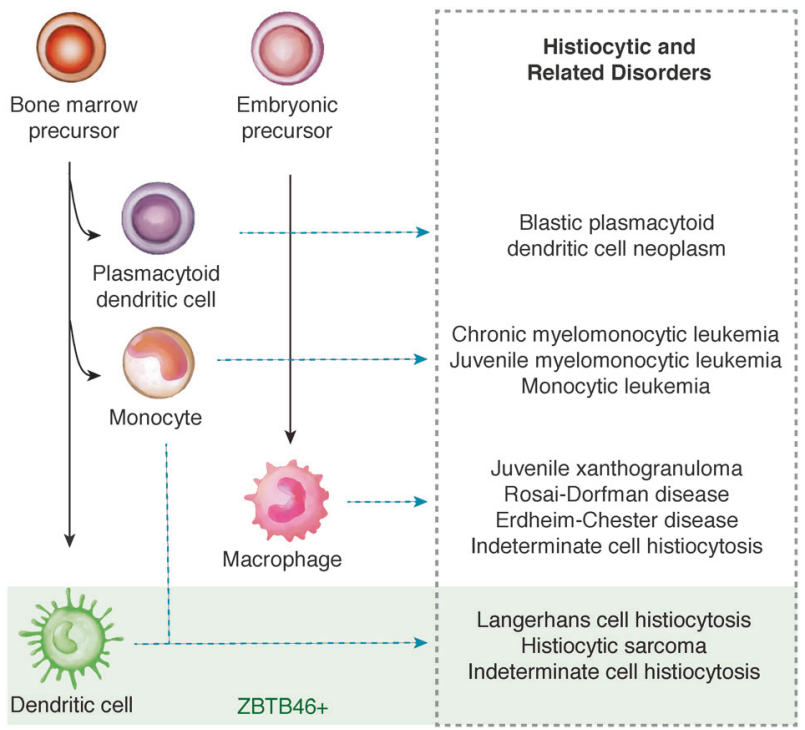

Fig. 3 Proposed schematic of cell identity of histiocytic disorders. The left columns indicate the development of normal human myeloid cell types, and the right column indicates the proposed classification of histiocytic disorders by cell identity based on the expression of ZBTB46

often show a hybrid ambiguous immunophenotypeexpressing both macrophage and dendritic cell markers [28-30]. Therefore, wider application of ZBTB46 immunohistochemistry to these entities may be diagnostically useful. Finally, evaluation of this marker in additional rare and poorly characterized diseases in the differential diagnosis for histiocytic neoplasms, such as interdigitating dendritic cell sarcoma and follicular dendritic cell sarcoma, may be informative. In particular, interdigitating dendritic cell sarcoma can share the exact morphologic and immunohistochemical profile as spindle cell melanoma, and differential expression of ZBTB46 in these two entities may have significant diagnostic utility [31].

More broadly, the use of ZBTB46 immunohistochemistry may aid in developing a classification scheme for histiocytic disorders that accurately reflects both cellular lineage and clinical behavior (Fig. 3), similar to that which exists for B- and T cell-derived cancers. Since dendritic cells are continually renewed from bone marrow progenitors, classification of Langerhans cell histiocytosis and histiocytic sarcoma as dendritic cell neoplasms supports their inclusion in the category of myeloproliferative disorders, as previously proposed [20], and may nominate alternative strategies for clinical treatment.

Acknowledgements This work was supported by the Stanford Pathology Department (to A.T.S., R.A.B., Y.N., and J.K.) and the Scleroderma Research Foundation (to H.Y.C.). A.T.S. was supported by a Parker Bridge Scholar Award from the Parker Institute for Cancer Immunotherapy.

\section{Compliance with ethical standards}

Conflict of interest The authors declare that they have no conflict of interest.

\section{References}

1. Satpathy AT, Wu X, Albring JC, Murphy KM. Re(de)fining the dendritic cell lineage. Nat Immunol. 2012;13:1145-54.

2. Satpathy AT, et al. Zbtb46 expression distinguishes classical dendritic cells and their committed progenitors from other immune lineages. J Exp Med. 2012;209:1135-52.

3. Meredith MM, et al. Expression of the zinc finger transcription factor $\mathrm{zDC}$ (Zbtb46, Btbd4) defines the classical dendritic cell lineage. J Exp Med. 2012;209:1153-65.

4. Satpathy AT, et al. Notch2-dependent classical dendritic cells orchestrate intestinal immunity to attaching-and-effacing bacterial pathogens. Nat Immunol. 2013;14:937-48.

5. Schreiber HA, et al. Intestinal monocytes and macrophages are required for $\mathrm{T}$ cell polarization in response to Citrobacter rodentium. J Exp Med. 2013;210:2025-39.

6. Wu X, et al. Mafb lineage tracing to distinguish macrophages from other immune lineages reveals dual identity of Langerhans cells. J Exp Med. 2016;213:2553-65.

7. Broz ML, et al. Dissecting the tumor myeloid compartment reveals rare activating antigen-presenting cells critical for $\mathrm{T}$ cell immunity. Cancer Cell. 2014;26:638-52.

8. Franklin RA, et al. The cellular and molecular origin of tumorassociated macrophages. Science. 2014;344:921-5.

9. Grajales-Reyes GE, et al. Batf3 maintains autoactivation of Irf8 for commitment of a $\mathrm{CD} 8 \alpha(+)$ conventional DC clonogenic progenitor. Nat Immunol. 2015;16:708-17.

10. Wang J, et al. Characterisation of ZBTB46 and DC-SCRIPT/ ZNF366 in rainbow trout, transcription factors potentially involved in dendritic cell maturation and activation in fish. Dev Comp Immunol. 2016;80:2-14.

11. Segura E, et al. Human inflammatory dendritic cells induce Th17 cell differentiation. Immunity. 2013;38:336-48.

12. Emile J-F, et al. Revised classification of histiocytoses and neoplasms of the macrophage-dendritic cell lineages. Blood. 2016;127:2672-81.

13. Arber DA, et al. The 2016 revision to the World Health Organization classification of myeloid neoplasms and acute leukemia. Blood. 2016;127:2391-405.

14. Gomez Perdiguero E, et al. Tissue-resident macrophages originate from yolk-sac-derived erythro-myeloid progenitors. Nature. 2015;518:547-51.

15. Fernandez-Pol S, Bangs CD, Cherry A, Arber DA, Gratzinger D. Two cases of histiocytic sarcoma with BCL2 translocations and occult or subsequent follicular lymphoma. Hum Pathol. 2016;55:39-43.

16. Hruz T, et al. Genevestigator v3: a reference expression database for the meta-analysis of transcriptomes. Adv Bioinforma. 2008;2008:420747.

17. Allen CE, et al. Cell-specific gene expression in Langerhans cell histiocytosis lesions reveals a distinct profile compared with epidermal Langerhans cells. J Immunol. 2010; 184:4557-67.

18. Nezelof C, Basset F, Rousseau MF. Histiocytosis X histogenetic arguments for a Langerhans cell origin. Biomedicine. 1973;18:365-71.

19. Berres M-L, et al. BRAF-V600E expression in precursor versus differentiated dendritic cells defines clinically distinct LCH risk groups. J Exp Med. 2015;212:281. 
20. Collin M, Bigley V, McClain KL, Allen CE. Cell(s) of origin of langerhans cell histiocytosis. Hematol Oncol Clin North Am. 2015;29:825-38.

21. Hogstad B, et al. RAF/MEK/extracellular signal-related kinase pathway suppresses dendritic cell migration and traps dendritic cells in Langerhans cell histiocytosis lesions. J Exp Med. 2018;215:319-36.

22. Swerdlow SH, et al. The 2016 revision of the World Health Organization classification of lymphoid neoplasms. Blood. 2016;127:2375-90.

23. Go $\mathrm{H}$, et al. Frequent detection of $\mathrm{BRAF}(\mathrm{V} 600 \mathrm{E})$ mutations in histiocytic and dendritic cell neoplasms. Histopathology. 2014;65:261-72.

24. Castro ECC, et al. Clinicopathologic features of histiocytic lesions following ALL, with a review of the literature. Pediatr Dev Pathol. 2010;13:225-37.

25. O'Malley DP, et al. Co-occurrence of Langerhans cell histiocytosis and Rosai-Dorfman disease: possible relationship of two histiocytic disorders in rare cases. Mod Pathol. 2010;23:1616-23.
26. Wood GS, Hu CH, Beckstead JH, Turner RR, Winkelmann RK. The indeterminate cell proliferative disorder: report of a case manifesting as an unusual cutaneous histiocytosis. J Dermatol Surg Oncol. 1985;11:1111-9.

27. Brown RA, et al. ETV3-NCOA2 in indeterminate cell histiocytosis: clonal translocation supports sui generis. Blood. 2015;126:2344-5.

28. Feldman AL, et al. Clonally related follicular lymphomas and histiocytic/dendritic cell sarcomas: evidence for transdifferentiation of the follicular lymphoma clone. Blood. 2008;111:5433-9.

29. Shao $\mathrm{H}$, et al. Clonally related histiocytic/dendritic cell sarcoma and chronic lymphocytic leukemia/small lymphocytic lymphoma: a study of seven cases. Mod Pathol. 2011;24:1421-32.

30. West DS, et al. Clonally related follicular lymphomas and Langerhans cell neoplasms: expanding the spectrum of transdifferentiation. Am J Surg Pathol. 2013;37:978-86.

31. Stowman AM, Mills SE, Wick MR. Spindle cell melanoma and interdigitating dendritic cell sarcoma: do they represent the same process? Am J Surg Pathol. 2016;40:1270-9.

\section{Affiliations}

\section{Ansuman T. Satpathy $\mathbb{D}^{1,2} \cdot$ Ryanne A. Brown ${ }^{1} \cdot$ Ellen Gomulia ${ }^{1} \cdot$ Carlos G. Briseño ${ }^{3}$ - Maxwell R. Mumbach ${ }^{2,4}$. Zenggang Pan $^{5} \cdot$ Kenneth M. Murphy ${ }^{3} \cdot$ Yasodha Natkunam ${ }^{1} \cdot$ Howard Y. Chang ${ }^{2,6} \cdot$ Jinah Kim $^{1}$}

1 Department of Pathology, Stanford University School of Medicine, Stanford, CA 94305, USA

2 Center for Personal Dynamic Regulomes, Stanford University School of Medicine, Stanford, CA 94305, USA

3 Department of Pathology and Immunology and Howard Hughes Medical Institute, Washington University School of Medicine, St. Louis, MO 63110, USA
4 Department of Genetics, Stanford University School of Medicine, Stanford, CA 94305, USA

5 Department of Pathology, University of Colorado Denver, Aurora, CO 80045, USA

6 Department of Dermatology, Stanford University School of Medicine, Stanford, CA 94305, USA 